\begin{tabular}{|c|c|}
\hline Title & Extraction of 3D vortex structures from a turbulent puff in a pipe using two-color illumination and flakes \\
\hline Author(s) & Ohkubo, Jumpei; Tasaka, Y uji; Park, Hy un Jin; Murai, Y uichi \\
\hline Citation & $\begin{array}{l}\text { Journal of visualization, 19(4), } 643-651 \\
\text { https://doi.org/10.1007/\$12650-016-0344 z }\end{array}$ \\
\hline Issue Date & 2016-11 \\
\hline Doc URL & http:/hdl.handle.net/2115/67494 \\
\hline Rights & The original publication is available at www.springerlink.com \\
\hline Type & article (author version) \\
\hline File Information & JOV_ohkubo_ver13rev.pdf \\
\hline
\end{tabular}

Instructions for use 


\title{
Extraction of 3D vortex structures from a turbulent puff in a pipe using two-color illumination and flakes
}

\author{
Jumpei Ohkubo, Yuji Tasaka*, Hyun Jin Park, Yuichi Murai \\ Laboratory for Flow Control, Faculty of Engineering, Hokkaido University, N13-W8, Sapporo 060-8628, Japan \\ *Corresponding author: E-mail: tasaka@eng.hokudai.ac.jp, Tel: +81-11-7066371
}

\begin{abstract}
A novel visualization technique was proposed to extract the three-dimensional vortex structure of a turbulent puff, which is a local turbulence event that is observed in pipe flows at relatively low Reynolds numbers. The technique is based on multi-color illumination of microscopic flakes that are suspended in the flow, which makes structural visualization more informative than conventional monochrome approaches. A special optical arrangement of two laser sheets, colored green and blue, was established for the circular pipe. Based on an image analysis sequence, the internal structure of the puff is reconstructed as a cross-sectional temporal 3D image consisting of voxels with unicolor degrees between green and blue, where an individual single vortex is extracted as a pair of two-color stripes. This allows quantification of the azimuthal wavenumber of the vortical structure that characterizes the puff. The wavenumber results agreed well with the results of previous studies, thus supporting the applicability of the proposed visualization technique.
\end{abstract}

Keywords: Pipe flow, Turbulence transition, Flow visualization, Flake

\section{Introduction}

The turbulent flow transition in a pipe is one of the most fundamentally important unsolved problems that remain in fluid mechanics after the epoch-making study of Osborn Reynolds (Reynolds 1883). Reynolds indicated that this transition is dominated by a non-dimensional number, the so-called Reynolds number $R e(=U D / v$, where $U$ is the mean velocity, $D$ is the pipe diameter and $v$ is the kinematic viscosity of the fluid); the flow has a local turbulence that consists of vortical structures that occur around a critical Reynolds number, $R e_{\mathrm{c}} \sim 2000$. In addition, $R e_{\mathrm{c}}$ can be increased dramatically to more than $10^{4}$ in more sophisticated flow facilities by reducing the inlet noise (more careful treatments of facilities push the critical Reynolds number up to $10^{5}$, Pfenniger 1961). This is because Hagen-Poiseuille flows are stable for infinitesimal perturbations for finite range of $\operatorname{Re}$ (e.g., Drazin 2002), and thus the transition occurs from finite amplitude perturbations or imperfection of facilities. Nowadays $R e_{\mathrm{c}} \sim 2000$ has been used in practical situations of engineering. Such subcritical instability problem is a common problem in wall-bounded shear flows, namely, boundary layers, Poiseuille and Couette flow families. More recent statistical studies on the creation and advection of a localized turbulence called turbulent puff (Wignanski and Champagne 1973) in controlled pipe flow facilities have provided a physical insight into this phenomenon: Peixinho and Mullin 2006 mentioned that the lifetimes of puffs, and the persistence times of the advections, increase exponentially with increasing $R e$. Meanwhile, Hof et al. 2006 indicated that the variation of lifetime is super exponential: It means that a turbulent puff is fundamentally transient and has a finite lifetime, even at high values of $R e$. An increasing $R e$ also accompanies the splitting of a puff toward formation of a turbulent slug (Wignanski and Champagne 1973), where the localized turbulence then grows into persistent turbulence. Avila et al. 2011 redefined $R e_{\mathrm{c}}$ as the crossing point between the curves of the lifetime and the splitting time with respect to the $R e$ as $R e_{\mathrm{c}}=2040 \pm 10$. Their results summarizing series of experimental and numerical works also support finite lifetime of puffs. Nevertheless, Peixinho and Mullin 2006 indicated that the lifetime of a turbulent puff approaches infinity around $R e=1750$. The work of Tasaka et al. 2010, which used the same facility used by Peixinho and Mullin 2006, also confirmed that puffs are persistent for $R e$ values 
higher than $1,750 \pm 10$. However, there is a difference between the systems used in these studies to induce the flow in a pipe: the system in Peixinho and Mullin 2006 had a constant mass flux and one in Avila et al. 2011 was operated with a constant pressure gradient. The former can keep the Re exactly constant, regardless of the flow transition, but its pressure gradient fluctuates. Further, Peixinho and Mullin 2006 adopted a special procedure to evaluate the lifetime: A turbulent puff was created at $R e=1,900$ and thus setting $R e$ was decreased to a prescribed value in the range 1,580 1,740. There is thus the possibility that this difference in the system and the procedure could cause small differences in the vortical structures of the puffs, and thus the lifetime characteristics may change.

A turbulent puff has a coherent structure that consists of a pair of streamwise vortices and low speed streaks (Fasst and Eckhardt 2003), and this structure has been confirmed by numerical simulations and experiments (Hof et al. 2004). This structure is called a traveling wave (TW), and has specific types of wavenumber in the circumferential direction. TWs are known as structures that sustain turbulence, and thus we suspect that it is important to carefully solve the problem of the experimental diversity of puffs and its dependence on the flow system. To clarify this problem, we need to perform a statistical analysis of the vortical structure of puffs. However, advanced particle image velocimetry (PIV) systems such as stereo-PIV and tomographic PIV cannot address this problem effectively because of the narrow dynamic range of PIV, i.e., the magnitude of the velocity fluctuation component in a puff often falls within the error level relative to the main flow velocity.

Visualization using flakes is a classical but feasible alternative to velocimetry-based approaches, and has been used to observe various flow structures that are subject to distortion (e.g., Park et al. 1981; Dominguez-Lerma et al. 1985; Bandyopadhyay 1986; Samanta et al. 2011). Using this method, local shear flows can be grasped directly as brightness information. Recently, interpretations of flow patterns visualized by adding flakes to fluid motion have been investigated (Goto et al. 2011; Einarsson et al. 2015). In addition, a methodology to quantitatively visualize vortex structures using flakes and multi-color illumination was proposed (Thoroddsen and Bauer 1999). Park et al. 2014 obtained vortex structures in wall turbulence using aluminum flakes and two laser sheets with different colors. This methodology is suitable for statistical investigation of the diversity of puffs because of its efficiency for problem-solving in researches, its direct vortex structure extraction capability and its ease of handling in comparison with PIV systems. We apply this methodology using two-color illumination and flakes to the visualization of 3D vortical structures in a puff. In this paper, our ideas for the optical set-up and several of the sequences required in the image analysis process are explained step-by-step. Furthermore, the extracted vortical structures are evaluated with respect to their relationships with the TWs.

\section{Experimental setup and visualization methodology}

Figure 1 shows an experimental pipe flow facility used to demonstrate the proposed visualization methodology. The pipe, including the test section, is made from acrylic resin with an inner diameter $D$ of $30 \mathrm{~mm}$ and an overall length of $4,000 \mathrm{~mm}(\sim 133 D)$. The test fluid is tap water, which is stored in the upstream head tank. The water level in the tank is kept constant to maintain a constant pressure gradient by supplying water from a pump and discharges through a drain cylinder set within the tank. The pipe is connected to the tank through a bell-mouth that has quasi-hyperbolic shape with smooth contraction of the diameter from $75 \mathrm{~mm}$ to $D$ in $23 \mathrm{~mm}$. The flow is driven by the pressure difference between the inlet and the outlet of the pipe, which is released to the atmosphere. The flow rate is controlled by a downstream valve and is monitored using a flow meter mounted near the valve. The Re, which is determined by the flow rate, was fixed at 2,300, and the kinematic viscosity $v$ is calculated from the temperature of the water in the tank, which is measured using a mercury thermometer. To control the creation of a fully developed turbulent puff by disturbing the main flow, a thin orifice with an inner diameter of $25 \mathrm{~mm}$ is mounted at the pipe inlet after the bell-mouth. The orifice creates puffs intermittently under these conditions. In absence of the orifice, puffs are created at moderate positions in the pipe at this Re and many of them are not fully developed. The test section used for visualization is located $120 \mathrm{D}$ downstream from the inlet, where we can assume that the turbulent puffs will be fully developed. The test section has a rectangular water jacket to prevent light reflection and refraction on the pipe surface. For better recognition of the optical configuration and the imaging planes, we set our coordinates as $x$ in the downstream direction, $y$ in the vertical direction and $z$ in the horizontal direction, as shown in Fig. 1.

For the visualization flakes, Kalliroscope flakes (AQ-1000, Kalliroscope Co.) are mixed into the test water. 
These flakes are platelets of protein crystal structures and the typical dimensions of the platelets are $6 \times 30 \times 0.07 \mu \mathrm{m}$ (Park et al. 1981). Traditional aluminum flakes and recently developed $\mathrm{TiO}_{2}$-coated mica platelets are also applicable for this visualization process. The advantages of using the Kalliroscope flakes are that they are thinner than the other available flakes (some $\mu \mathrm{m}$ in both aluminum flakes and mica platelets), which enables them to visualize fine structures and provide better responses to fluctuations of the flows. And, their relatively low density $\left(1,620 \mathrm{~kg} / \mathrm{m}^{3}\right.$ for Kalliroscope, $2,700 \mathrm{~kg} / \mathrm{m}^{3}$ for aluminum flakes, and $2,800 \mathrm{~kg} / \mathrm{m}^{3}$ for mica platelets) prevents them from sinking in the pipe. Furthermore, the Kalliroscope flakes are soft, whereas the other platelets are hard and cause damage to the acrylic pipe wall.

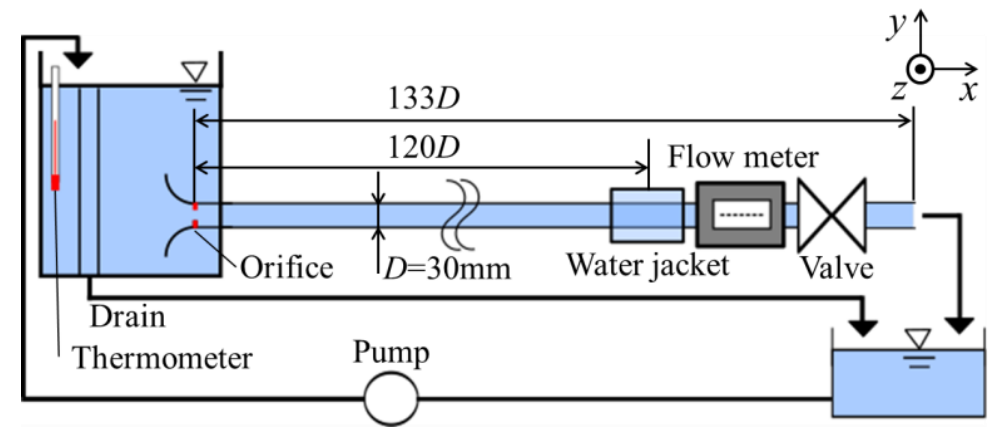

Fig. 1 Experimental facility

The flakes have orientation characteristics relative to the local shear directions of the flows and the light beams that are reflected from the flake surfaces, as shown in Fig. 2(a), form flow patterns on the visualized images according to the shear flow structures. Originally, this visualization technique using single light source provided local shear structures and not vortex structures. To distinguish the vortices in the images, we need either knowledge of the flow or additional information. We therefore adopt the two-laser sheet technique (Park et al. 2014). The principle for visualization of vortex structures was explained in depth in the work of Park et al. 2014, where the visualization objects are longitudinal vortices in the turbulent boundary layer of a horizontal channel flow; the two counter laser sheets were used to illuminate a horizontal plane near the channel wall. Unlike this work, the vortices in the puff were arranged in a circumferential direction and were skewed in the streamwise direction (Hof et al. 2004; Shimizu and Kida 2009). We thus cannot use the same optical configuration as that of Park et al. 2014, and must have an appropriate configuration for this geometry. Figure 2(b) shows the required orientation of the optical instruments to visualize the vortex structures in a puff via the illumination of the two laser sheets. A green laser (center wavelength $\lambda$ $=532 \mathrm{~nm})$ and a blue laser $(\lambda=473 \mathrm{~nm})$ were established facing each other. The intensities of both laser beams were adjusted to $100 \mathrm{~mW}$, and the laser sheets were formed using cylindrical lenses that were mounted in front of the lasers. The thickness of each sheet is approximately $2 \mathrm{~mm}$. The laser sheets both irradiated the same cross-section, which is inclined at $45^{\circ}$ with respect to the main flow direction, i.e., the $x$-axis, as shown in Fig. 2(b). The color image sequence of the visualized flow patterns was taken from the bottom of the pipe using a high-speed video camera (FASTCAM-MAX, Photron) that can capture color images using the complementary metal-oxide-semiconductor (CMOS) sensor in the camera imager, and these images are recorded as raw 8-bit color files. The frame rate settings were a frame rate of $125 \mathrm{fps}$ and a shutter speed of $1 / 125 \mathrm{~s}$. The image size is $512 \times 512$ pixels and the corresponding image resolution is $0.123 \mathrm{~mm} / \mathrm{pixel}$. When the different two-color lasers irradiate a vortex in the face-to-face configuration, the reflected light provides a pair of two-color stripes, as shown in Fig. 2(c). Each vortex can then be distinguished and extracted as a pair of stripes. 
(a)

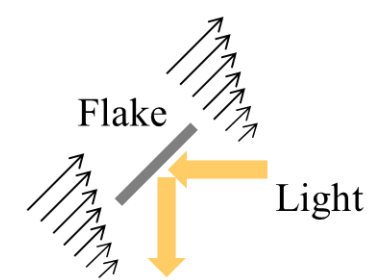

Shear flow (b)
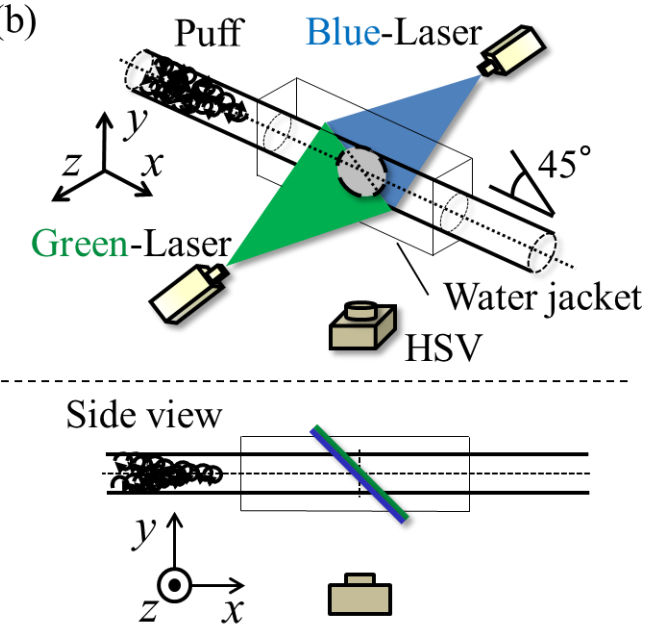

(c)

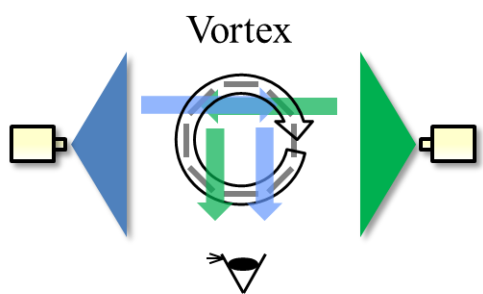

Fig. 2 Schema of the visualization principles: (a) light reflection characteristics of a flake in a flow, (b) arrangement of the optical instruments on the pipe facility, and (c) light reflection characteristics for a vortex

\section{Experimental results}

\subsection{Visualized image of a passing puff}

Figure 3 shows the original images of a puff passing the test section that were visualized using the two-color laser illumination system. Figure 3(a) shows a timeline image that was extracted at the horizontal center line of the pipe from sequential images; the image contains almost an entire picture of a single turbulent puff, where the flow structure is expressed through the contrast of the green, blue and black colors based on the optical characteristics of this technique, as explained in the previous section, and the reflections of the laser sheets at the pipe wall. Pointwise measurements of the velocity at the center of the pipe cross-section for a turbulent puff show a gradual decrease in velocity on the foreside, growth of fluctuations in the backward direction, and a sharp trailing edge (Wignanski and Champagne 1973). The visualized puff in the timeline image agrees with these features qualitatively, and the cuneiform profile shown in the cross-section is also one of the specific features of turbulent puffs (Darbyshire and Mullin 1995). The length of the puff, which was calculated using the passing time and the mean velocity, is $28.8 D$, which is close to the approximate value of $20 \mathrm{D}$ from a previous observation (Wignanski and Champagne 1973).

Figure 3(b) and (c) show snapshots of a passing turbulent puff, and Fig. 3(d) represents the laminar flow after the puff has passed. The corresponding times of these snapshots are indicated in the timeline image of Fig. 3(a) as white lines. Because of the tilt of the incident laser sheets relative to the horizontal plane, as shown in Fig. 2(b), the illuminated areas in the snapshots (Fig. 3(b)-(d)) contain brighter areas with different colors as quadrupole distributions; the incident laser sheets from the opposite direction are reflected at the inside and outside walls of the pipe in the direction of the camera, depending on the face of the wall curve. Images corresponding to shear flow structures, which are expressed as fluctuation patterns on the images, are superimposed on this quadrupole pattern. The laminar flow in this situation, which is represented by the parabolic-shaped velocity profile of the Hagen-Poiseuille flow, shows a large-scale organized pattern on the image with gradual contrasts of blue and green. However, the flow does not show the fine fluctuation patterns, and we can thus assume that Fig. 3(d) is an image of the undisturbed case; variations of the flow structure are extracted as differences relative to this base image. Figure 3(b) is a snapshot of the foreside of the puff, and only the central part of the section is disturbed. As the puff passes through, the disturbed part extends towards the pipe wall. At the rear region of the puff shown in Fig. 3(c), the entire section of the pipe is disturbed. 


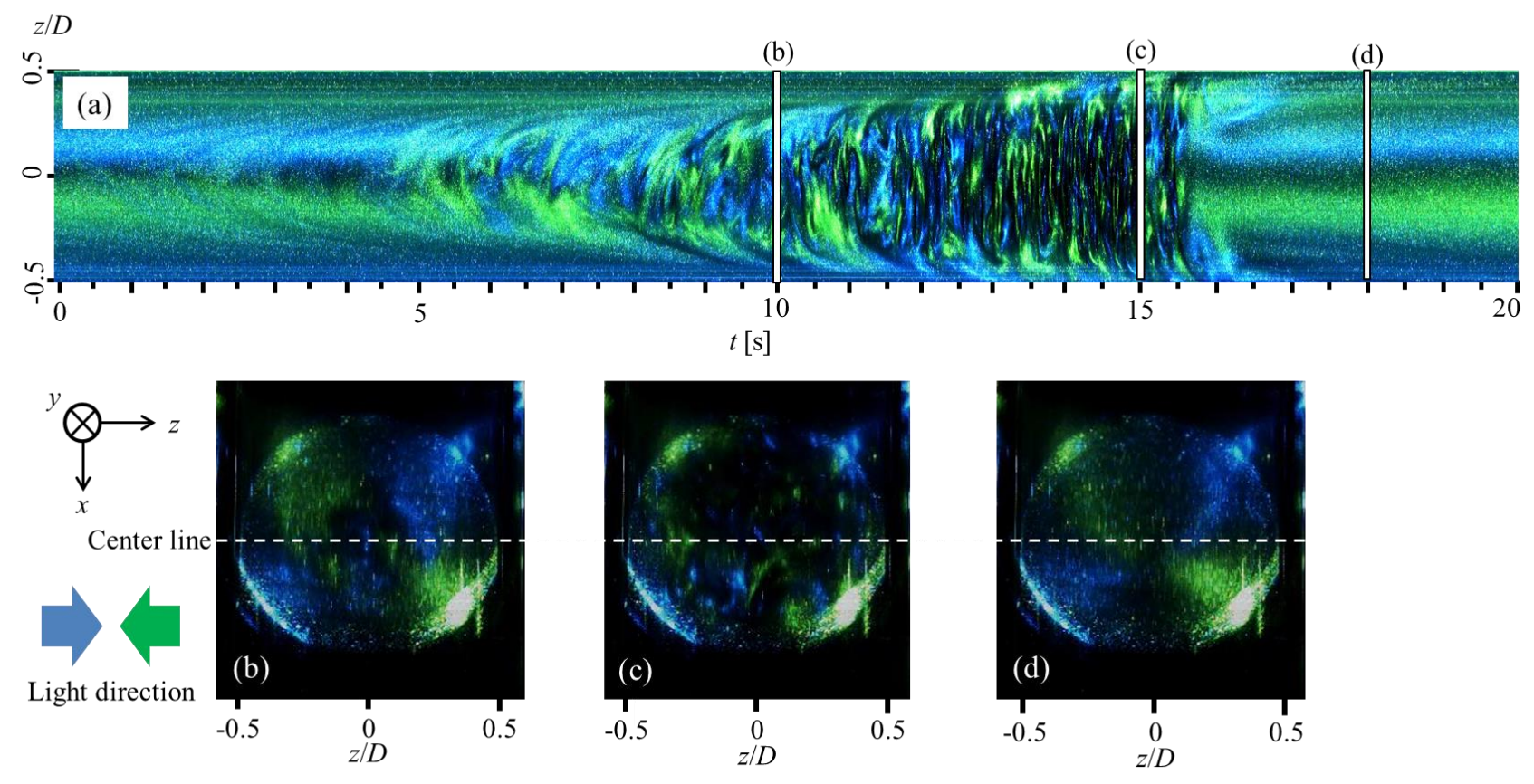

Fig. 3 Raw images of visualized flow: (a) timeline image extracted at the horizontal center line of the pipe from sequential images; snapshots of (b) the foreside region of the puff, (c) the rear, disturbed region of the puff, and (d) the laminar flow

\subsection{Image processing}

To extract the three-dimensional vortical structure of a turbulent puff, image processing is required, even though the original image obtained by the presented technique (as shown in Fig. 3) provides a quantitatively good representation of the structure. In this section, the details of the image processing steps are explained individually to illustrate the meaning of each process. The process consists of three steps: background removal, color adjustment, and inclination compensation. In an actual analysis, of course, all three steps can be performed as a single task in a very short time of approximately $300 \mathrm{~s}$, unlike the heavy processing required for PIV-based analyses.

3.2.1 Background removal: As explained in the previous section, the raw image of the flow that was visualized by the present technique contains three different information aspects: reflection at the wall, laminar base flow, and the vortex structure of the puff as expressed by the color patterns. The first process is, therefore, removal of the background image to extract the vortex structure. The time-averaged RBG intensity image in the laminar flow shown in Fig. 3(d) is used as the background image, where both the reflection pattern on the pipe surface and the color pattern that corresponds to the laminar flow are superimposed. Therefore, the residual of this background subtraction should correspond to the color pattern of the vortex structures. Figure 4 shows snapshots of the visualized flow pattern in the laminar flow, in the form of (a) a raw image, (b) a time-averaged color intensity image that was calculated from 200 images in the laminar flow, and (c) a residual image produced by subtraction of (b) from (a). The intensity in the residual images is the value of the raw image minus that of the background image at each pixel and each RGB component, where negative values that occur as a result of subtraction are replaced with zeros to remove uncertain information amplifying measurement errors. The scattered fine patterns on the residual image in the laminar case represent the degree of non-uniformity of the flakes at that moment. 

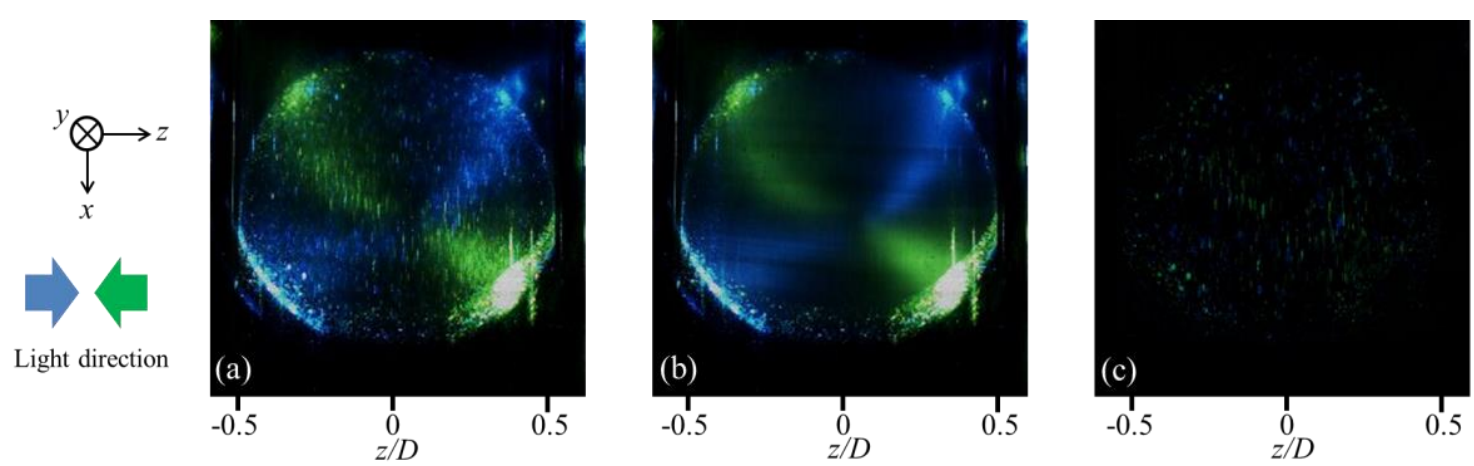

Fig. 4 Images in a background removal process: (a) raw, (b) background, and (c) residual images

3.2.2 Adjustment of color balance: General imaging devices have uneven sensitivities to the colors of incident light, even if each color component of the light has the same intensity. This is because these devices have been designed to represent the sensitivity of human eyes to colors. Furthermore, each CMOS element in the devices has broad sensitivity to the wavelengths of the light and the sensitive bands overlap each other (e.g., El Gamal and Eltoukhy 2005). For example, pure blue light is not recognized as a "perfect blue" by the sensing elements, because it will contain small green and red components. In the presented technique, the vortex structures can be recognized as color stripe patterns, and we must therefore adjust the color balance of the image evenly to prevent erroneous recognitions. The test fluid contains numerous flakes and each flake has the light reflection characteristics shown in Fig. 2(a). Fine multi-reflections occur between these flakes, and thus the corresponding color information of the vortex structure obtained from the incident two-color laser beams is captured as a probability distribution of the color intensities of blue $(B)$ and green $(G)$ at each pixel on these images. Suitable adjustment of the color balance then makes the extraction of the vortex structures possible according to their probability.

Here, we assume that the averaged values of $B$ and $G$ over the image should be the same. The averaged intensity of each color $\left(G_{\text {ave }}\right.$ and $\left.B_{\text {ave }}\right)$ is calculated from an 1800 image sequence with the passing of a puff. Then, $B$ for each pixel is multiplied by the ratio $G_{\text {ave }} / B_{\text {ave }}$ to equalize the levels of the green and blue intensities. Figure 5 shows an example of color adjustment, where Fig. 5(a) is a snapshot image before the adjustment and Fig. 5(b) is an image with the adjustment. After the adjustment of the color balance, the dominant color phase can be determined as a single color, i.e., green or blue, at each pixel on the image. The images taken using the high-speed video camera also have the intensity of a red color component $(R)$ in addition to $B$ and $G$. In this unicolor adjustment, $R$ is replaced by a zero at every pixel. Then, the dominant color is determined to be either blue or green via a comparison between $B$ ' and $G$. The actual processing involves the intensity at each pixel being changed into the difference between green and blue, i.e., $G^{\prime}$ $=G-B^{\prime}$ for cases where $G>B^{\prime}$, and $B^{\prime \prime}=B^{\prime}-G$ for $B^{\prime}>G$. Goto et al. 2011 mentioned that strong reflections are obtained, regardless of the light direction, because of the isotropic orientation of the flakes. This unicolor adjustment is equivalent to the extraction of the anisotropic flow structures like vortices. Figure 5(c) shows an example of the unicolor adjustment of the image shown in Fig. 5(b).
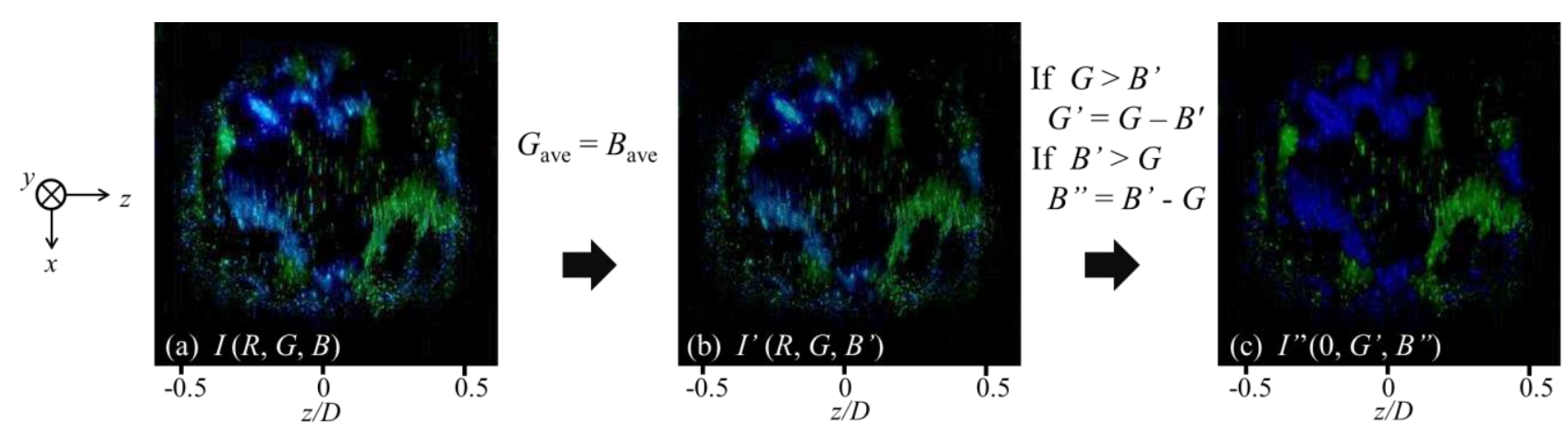

Fig. 5 Color adjustment: (a) image without the adjustment, (b) image with the color balance adjustment, and (c) image with the unicolor adjustment at each pixel $(t \sim 15.6 \mathrm{~s}$ in Fig. 3(a)) 
3.2.3 Compensation of illumination inclination: In the present configuration, the sheet-shaped illumination is irradiated at an angle of $45^{\circ}$ relative to the normal pipe cross-section to observe the whole of the pipe section. Therefore, the color information obtained that represents the vortex structure is on the inclined cross-section shown in Fig. 2(b), and we must rearrange the pixels to reconstruct the normal cross-sections at each moment. Figure 6(a) shows the arrangement of the two color laser sheets used to irradiate the pipe flow. A gap of $a(h)$ between the imaging plane and the normal cross-section of the pipe is dependent on the height $h$ from the centerline of the pipe in the form $a(h)=$ $h$. We assume that the vortex structure inside the puffs remains unchanged over the short time that puffs take to pass through the measurement section, and we thus perform a temporal rearrangement of the images based on this frozen hypothesis. The local velocity is not uniform in the pipe cross-section, but the traveling speed of puffs including inner vortical structures is constant. The traveling time of a puff passing through the gap $a(h)$, denoted by $t_{\mathrm{p}}$, is derived from the puff advection speed $V$ in the form $t_{\mathrm{p}}(h)=a(h) / V$. In the calculations, $V=0.9 U$ is used, based on the work of Hof $e t$ al. 2005, where it was indicated that $V$ is dependent on the Reynolds number. This value is also confirmed in the present experiment via tracking of flow patterns on visualized images. The horizontal pixel lines in the image are rearranged in the time direction and the corresponding number of frames that must be shifted is determined from $t$, the frame rate, and $a(h)$, where the maximum number of shifted frames is 22. Figure 6(b) and (c) show the images before and after compensation. The sequence of reconstructed images now represents the vortex structure of a puff passing through the set cross-section of the pipe.

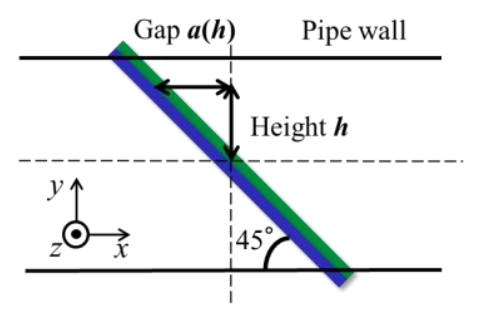

(a)
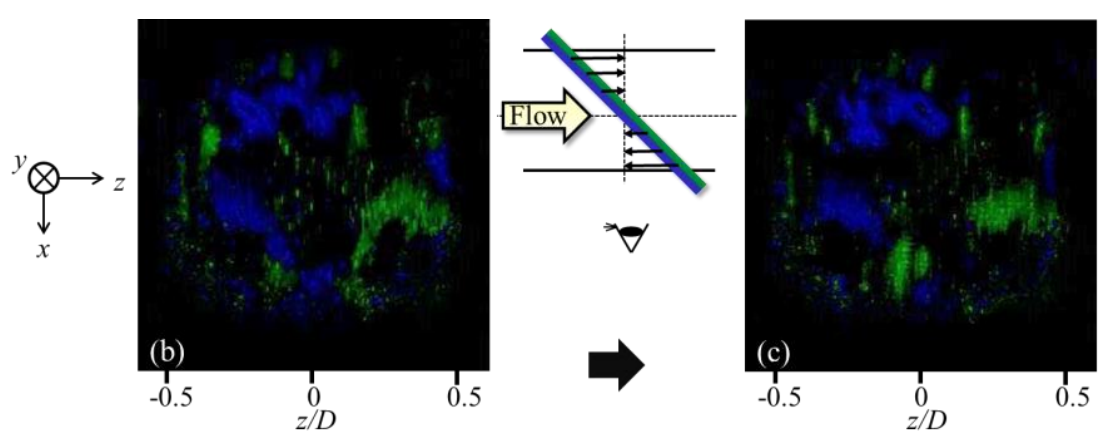

Fig. 6 (a) Optical configuration and inclined cross-section (the two laser sheets are overlapping), and images (b) before and (c) after compensation of the section inclination

\subsection{Extraction of the vortex structure}

Following the image processing sequence described above, the vortex structures are displayed as color stripes consisting of voxels with unicolor information in $y, z$, and $t$ coordinates. Figure 7 shows examples of reconstructed three-dimensional structures of the vortices in a puff from different viewpoints. The figure shows that the stripes are skewed gently on the foreside of the puff, but are hardly skewed at all in the backward region of the puff. The TW structure is thought to be located around the trailing edge of the puff, where the turbulence region occupies the entire pipe cross-section, which is in the time range from 13 to $16 \mathrm{~s}$ in Fig. 7(a). Stripe patterns with slopes are observed in the reconstructed image, and these patterns may indicate the wavy structure that was described by Hof et al. 2005.

Figure 8(a) shows the timeline image in the vicinity of the pipe's inner wall, where $r / D=0.47$ is extracted from the voxels, and where a median filter was used to remove irregular noise. The time range displayed in the graph is from 13 to $16 \mathrm{~s}$, where the turbulence is at its most active in the puff. As described in Sec. 2, a streamwise vortex is extracted as a pair of two-color stripes. In Fig. 8(a), there are some pairs of two-color stripe patterns, and we can distinguish the numbers, lengths and slopes of the vortices from the figure. The TW consist of at least a pair of two vortices, so the wavenumber of the TW is estimated from the number of vortices along the circumferential direction. In Fig. 8(a), approximately four pairs of stripe patterns are observed along the circumferential direction, which means that two longitudinal vortices exist in that half of the circumference. Based on the variation of the traveling speed of the puffs, the wavenumber of each TW changes depending on the Re. Hof et al. 2004 indicated that the corresponding number of waves in the circumferential direction for the present Reynolds number $(R e=2,300)$ is four, which is equal to the value determined by the present estimation method. Figure $8(\mathrm{~b})$ represents vortex structure at slightly inside 
layer at $r / D=0.43$. The structure is still visible around the trailing edge, but becomes unclear toward middle part of the puff.

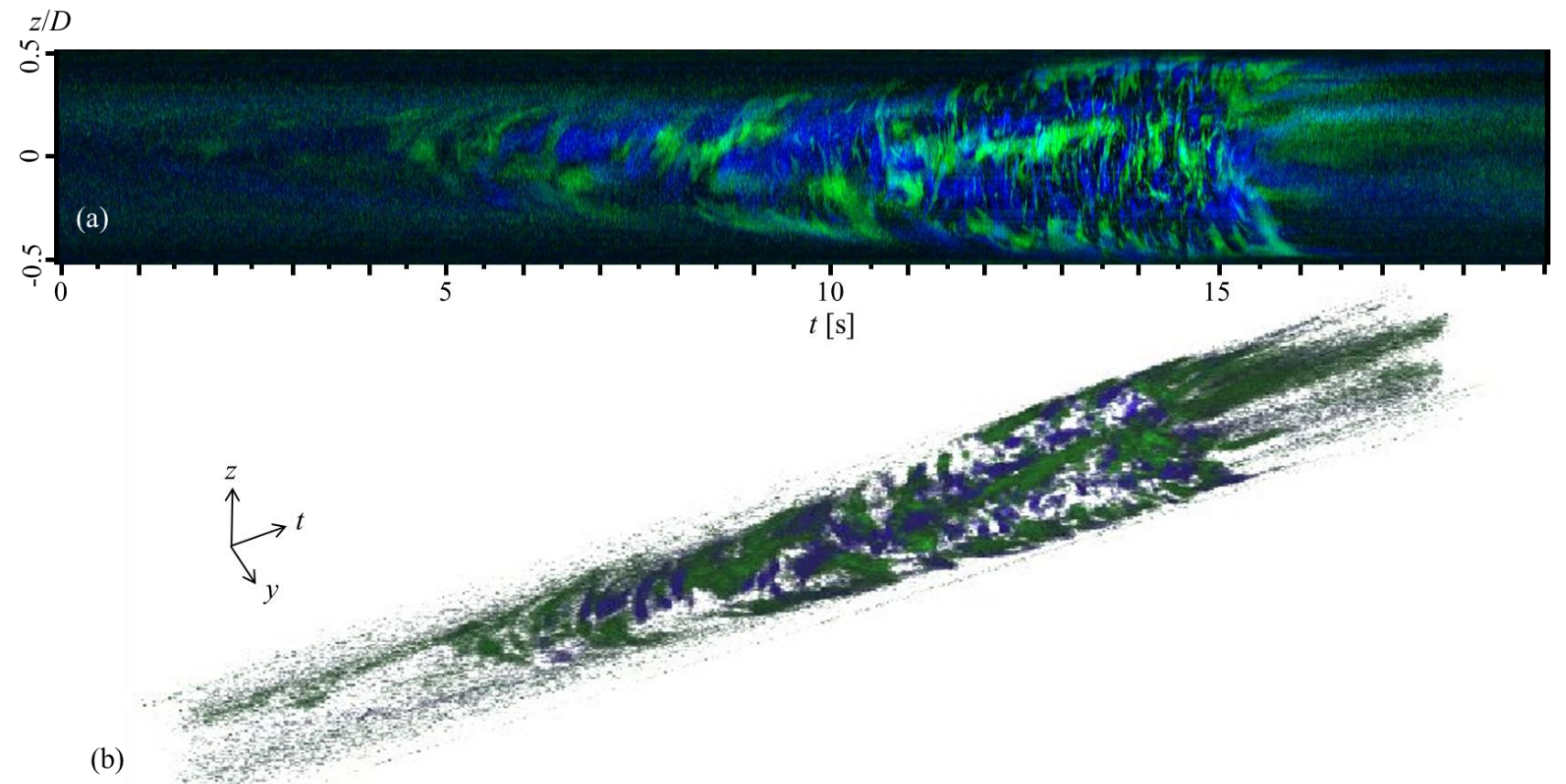

Fig. 7 Three-dimensional vortex structure of a turbulent puff represented by color stripes, (a) side view and (b) birds-eye view

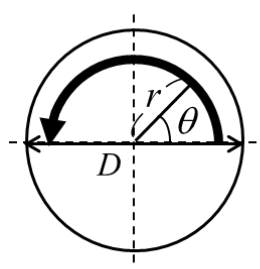

(a) $\theta[\mathrm{rad}]$

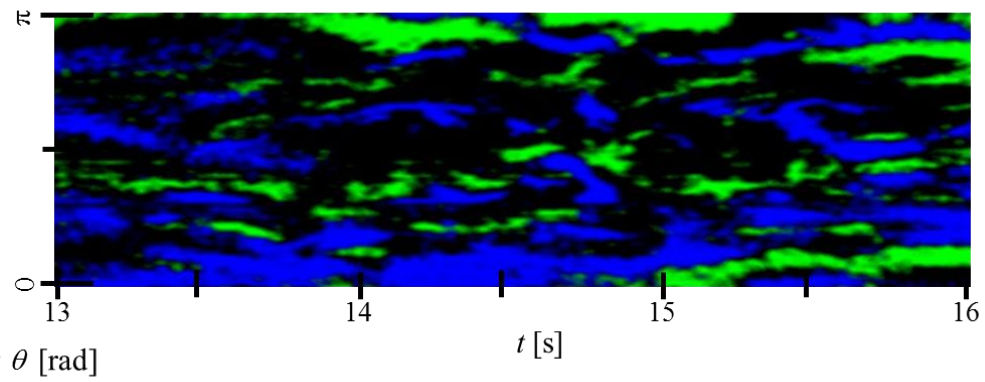

(b)

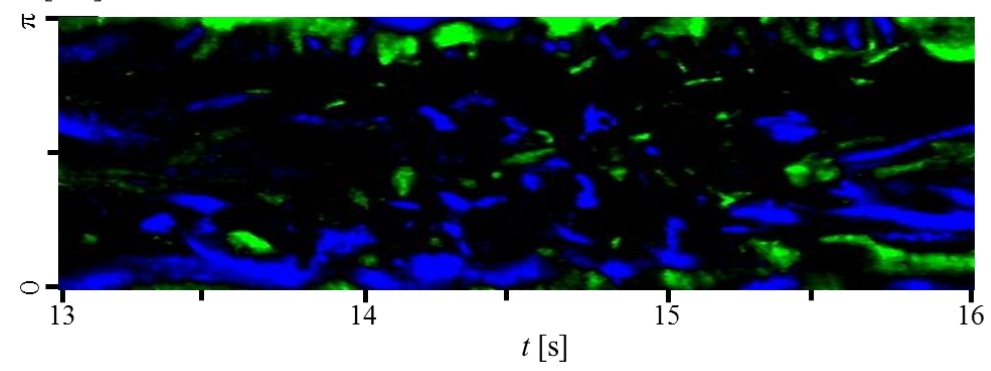

Fig. 8 Azimuthal-temporal distribution of color information, representing vortices extracted in the vicinity of the pipe's inner wall, (a) $r / D=0.47$ and (b) $r / D=0.43$, where pairs of two-color streaks indicate a single streamwise vortex formed inside the puff

\section{Conclusions}

This study proposed a novel visualization technique using flakes to extract the three-dimensional vortex structures from a turbulent puff, which is a local turbulence event that appears in pipe flows, without performing velocity field measurements. Two-color (blue and green) laser sheets, which were arranged to be facing each other and tilted relative 
to the normal cross-section of the pipe, represented the vortex structures as colored stripes. A sequence involving subtraction of the laminar flow background image, adjustment of the color information and rearrangement of the images to a normal cross-section was used to achieve extraction of the three-dimensional vortex structure as voxels with unicolor information. The number of vortices at the trailing edge of the puff that was estimated from the reconstructed 3D vortex structure agrees with the values obtained in previous works.

The sequence of image processing summarized above does not require large computer resource and is not time-consuming in comparison with velocity field measurement based approaches. This advantage allows us for doing various trials to explore hidden physics under the complex fluid motions other than the measurement of longitudinal vortices stated here. For example, tracking the color voxel patterns representing longitudinal vortices may elucidate advection characteristics of the vortices efficiently.

\section{Acknowledgments}

This study was supported by JSPS KAKENHI through Grant No. 25420103. The authors would like to express their thanks for this support.

\section{References}

Avila K, Moxey D, Lozar A, Avila M, Barkley D, Hof B (2011) The Onset of Turbulence in Pipe Flow. Science 333:192-196. doi:10.1126/science.1203223

Bandyopadhyay PR (1986) Aspects of the equilibrium puff in transitional pipe flow. Journal of Fluid Mechanics 163:439-458

El Gamal A, Eltoukhy H (2005) CMOS image sensors: An introduction to the technology, design, and performance limits, presenting recent developments and future directions. IEEE Circuits Devices Magazine 21:6-20. doi:10.1109/MCD.2005.1438751

Darbyshire AG, Mullin T (1995) Transition to turbulence in constant-mass-flux pipe flow. Journal of Fluid Mechanics 289:83-114.

Dominguez-Lerma MA, Ahlers G, Channell DS (1985) Effects of "Kalliroscope" flow visualization particles on rotating Couette-Taylor flow. Physics of Fluids 28:1204-1206.

Drazin PG (2002) Introduction to hydrodynamic stability, Cambridge University Press.

Einarsson J, Candelier F, Lundell F, Angilella JR, Mehlig B (2015) Rotation of a spheroid in a simple shear at small Reynolds number. Physics of Fluid 27:063301. doi:10.1063/1.4921543

Faisst H, Eckhardt B (2003) Traveling Waves in Pipe Flow. Physical Review Letters 91:224502. doi:10.1103/PhysRevLett.91.224502

Goto S, Kida S, Fujiwara S (2011) Flow visualization using reflective flakes. Journal of Fluid Mechanics 683:417-429. doi:10.1017/jfm.2011.299

Hof B, Doorne C, Westerweel J, Nieuwstadt F, Faisst H, Eckhardt B, Wedin H, Kerswell R, Waleffe F (2004) Experimental Observation of Nonlinear Traveling Waves in Turbulent Pipe Flow. Science 305:1594-1597. doi: $10.1126 /$ science. 1100393

Hof B, Westerweel J, Schneider TM, Eckhardt B (2006) Finite lifetime of turbulence in shear flows. Nature 443:59-62. doi:10.1038/nature05089

Hof B, Doorne C, Westerweel J, Nieuwstadt F (2005) Turbulent Regeneration in Pipe Flow at Moderate Reynolds Numbers. Physical Review Letters 95:214502. doi:10.1103PhysRevLett.95.214502

Park K, Grawford GL, Donnelly RJ (1981) Determination of Transition in Couette Flow in Finite Geometries. Physical Review Letters 47: 1448-1450.

Park HJ, Tasaka Y, Murai Y, Oishi Y (2014) Vortical structures swept by a bubble swarm in turbulent boundary layers. Chemical Engineering Science 116:486-496.

Peixinho J, Mullin T (2006) Decay of Turbulence in Pipe Flow. Physical Review Letters 96:094501. doi:10.1103/PhysRevLett.96.094501

Pfenniger W (1961) Transition in the inlet length of tubes at high Reynolds numbers. Boundary layer and flow control (ed. GV Lachman):970-980.

Reynolds O (1883) An Experimental Investigation of the Circumstances which Determine whether the Motion of Water shall be Direct or Sinuous, and of the Law of Resistance in Parallel Channels. Philosophical Transactions of the Royal Society of London 35:84-99.

Samanta D, Lozar A, Hof B (2011) Experimental investigation of laminar turbulent intermittency in pipe flow. Journal of Fluid Mechanics 681:193-204. doi:10.1017/jfm.2011.189 
Shimizu M, Kida S (2009) A driving mechanism of a turbulent puff in pipe flow. Fluid Dynamics Research 41:045501. doi:10.1088/0169-5983/41/4/045501

Tasaka Y, Schneider TM, Mullin T (2010) Folded Edge of Turbulence in a Pipe. Physical Review Letters 105:174502. doi:10.1103/PhysRevLett.105.174502

Thoroddsen ST, Bauer JM (1999) Qualitative flow visualization using colored lights and reflective flakes. Physics of Fluids 11:1702-1704.

Wignanski IJ, Champagne FH (1973) On transition in a pipe. Part 1. The origin of puffs and slugs and the flow in a turbulent slug. Journal of Fluid Mechanics 59:281-335. 